TAIWANESE JOURNAL OF MATHEMATICS

Vol. 15, No. 1, pp. 365-380, February 2011

This paper is available online at http://www.tjm.nsysu.edu.tw/

\title{
ON VECTOR EQUILIBRIUM PROBLEMS AND APPLICATIONS
}

\author{
Adela Capătă and Gábor Kassay
}

\begin{abstract}
The main purpose of this paper is the study of sufficient conditions for the existence of solutions of vector equilibrium problems. Our main results permit to obtain some existence results already established for the scalar case and, to provide some applications in the area of vector optimization and vector saddle point problems.
\end{abstract}

\section{INTRODUCTION}

Let $A$ and $B$ be two nonempty sets and $\varphi: A \times B \rightarrow \mathbb{R}$ a given function. A problem which has been extensively studied in the recent literature (e.g., $[1-3,5,8$, $11])$ is the so called scalar equilibrium problem:

(EP) find $\bar{a} \in A$ such that $\varphi(\bar{a}, b) \geq 0$, for all $b \in B$.

It is well-known (see for instance [5]) that this problem contains, in particular, optimization problems, variational inequalities, saddle point problems, Nash equilibrium, and other problems of interest in many practical applications. If the scalar function $\varphi$ is replaced by a vector-valued function, say $\varphi: A \times B \rightarrow Z$, where $Z$ is a topological vector space, partially ordered by a convex cone $C \subseteq Z$ with $\operatorname{int} C \neq \emptyset$, one may consider the so-called vector equilibrium problem in two ways:

(VEP) find $\bar{a} \in A$ such that $\varphi(\bar{a}, b) \notin-C \backslash\{0\}$, for all $b \in B$, and

(WVEP) find $\bar{a} \in A$ such that $\varphi(\bar{a}, b) \notin-\operatorname{int} C$, for all $b \in B$.

The first problem is called (strong) vector equilibrium problem, while the second one is called weak vector equilibrium problem.

Received September 5, 2008, accepted July 17, 2009.

Communicated by F. Giannessi.

2000 Mathematics Subject Classification: 90C47, 49J35.

Key words and phrases: Vector equilibrium problem, Cone-lower semicontinuity, Subconvexlike function, Cone-saddle point.

Research supported by the Grant PN II, ID 523/2007. 
Vector equilibrium problems are natural extensions of several problems of practical interest like vector optimization problems or vector variational inequality problems.

The aim of this paper is to provide sufficient conditions for the existence of solutions of (WVEP) in a general setting, and to apply the results for vector optimization and vector saddle point problems. The paper is organized as follows. In section 2 we present our main result (Theorem 2.4) which is based on a separation theorem of two disjoint convex sets in infinite dimensional topological vector spaces. This theorem is a generalization to the vector case of a scalar result proved in [10]. The last two sections are devoted to applications. In section 3 we apply our existence results for vector optimization problems, while in section 4 we established a so-called cone-saddle point theorem, related to a concept of saddle point for vector-valued functions introduced in [14].

\section{Sufficient Conditions for Weak Solutions}

Let us recall some concepts and properties needed in this sequel. Let $E$ be a topological space and $Z$ be a real topological vector space such that $Z$ is partially ordered by a convex cone $C$ with $\operatorname{int} C \neq \emptyset$, i.e., for any $z_{1}, z_{2} \in Z, z_{1} \leq_{C} z_{2}$ iff $z_{2}-z_{1} \in C$.

Let $Z^{*}$ be the dual space of $Z$, and let $C^{*}$ be the dual cone of $C$, i.e. $C^{*}=$ $\left\{z^{*} \in Z^{*} \mid z^{*}(k) \geq 0\right.$ for all $\left.k \in C\right\}$.

Definition 2.1. [7]. A function $f: E \rightarrow Z$ is said to be $C$-upper semicontinuous at $x \in E$ ( $C$-usc in short) if it satisfies the following condition:

$1^{\circ}$ For any neighborhood $V_{f(x)} \subset Z$ of $f(x)$, there exists a neighborhood $U_{x} \subset$ $E$ of $x$ such that $f(u) \in V_{f(x)}-C$ for all $u \in U_{x}$.

Remark 2.2. By [14] condition $1^{\circ}$ from the above definition is equivalent to:

$2^{\circ}$ For any $k \in \operatorname{int} C$, there exists a neighborhood $U_{x} \subset E$ of $x$ such that $f(u) \in f(x)+k-\operatorname{int} C$ for all $u \in U_{x}$.

Notice that in [7], this notion was termed $-C$-continuous function at $x$. The function $f$ is said to be $C$-lower semicontinuous ( $C$-lsc in short) if $-f$ is $C$-usc.

Recall the following well-known property, which is easy to prove.

Lemma 2.3. If $z^{*} \in C^{*}$ is a nonzero functional, then $z^{*}(z)>0$ for all $z \in$ int $C$.

Let $A$ be a nonempty subset of $E, B$ a nonempty set, and let $\varphi: A \times B \rightarrow Z$. We study the following weak vector equilibrium problem:

(WVEP) $\quad$ find $\bar{a} \in A$ such that $\varphi(\bar{a}, b) \notin-\operatorname{int} C$, for all $b \in B$. 
The next result provides sufficient conditions for the existence of solutions of (WVEP).

Theorem 2.4. Let $A$ be a compact set and let $\varphi: A \times B \rightarrow Z$ be a function such that

(i) for each $b \in B$, the function $\varphi(\cdot, b): A \rightarrow Z$ is $C$-usc on $A$;

(ii) for each $a_{1}, \ldots, a_{m} \in A, \lambda_{1}, \ldots, \lambda_{m} \geq 0$ with $\sum_{i=1}^{m} \lambda_{i}=1, b_{1}, \ldots, b_{n} \in B$ there exists $u^{*} \in C^{*} \backslash\{0\}$ such that

$$
\min _{1 \leq j \leq n} \sum_{i=1}^{m} \lambda_{i} u^{*}\left(\varphi\left(a_{i}, b_{j}\right)\right) \leq \sup _{a \in A} \min _{1 \leq j \leq n} u^{*}\left(\varphi\left(a, b_{j}\right)\right) ;
$$

(iii) for each $b_{1}, \ldots, b_{n} \in B, z_{1}^{*}, \ldots, z_{n}^{*} \in C^{*}$ not all zero one has

$$
\sup _{a \in A} \sum_{j=1}^{n} z_{j}^{*}\left(\varphi\left(a, b_{j}\right)\right) \geq 0 .
$$

Then the equilibrium problem (WVEP) admits a solution.

Proof. Suppose by contradiction that (WVEP) has no solution, i.e. for each $a \in A$ there exists $b \in B$ with the property $\varphi(a, b) \in-\operatorname{int} C$. This means that for each $a \in A$ there exist $b \in B$ and $k \in \operatorname{int} C$ such that

$$
\varphi(a, b)+k \in-\operatorname{int} C .
$$

Consider the sets

$$
U_{b, k}:=\{a \in A \mid \varphi(a, b)+k \in-\operatorname{int} C\},
$$

where $b \in B$ and $k \in \operatorname{int} C$. In what follows we show that the family of these sets forms an open covering of the compact set $A$.

Let $a_{0} \in U_{b, k}$ and $k \in \operatorname{int} C$. Since $a_{0} \in U_{b, k}$ we have that

$$
\varphi\left(a_{0}, b\right)+k \in-\operatorname{int} C \text { that is, }-\varphi\left(a_{0}, b\right)-k \in \operatorname{int} C .
$$

Denote $k^{\prime}:=-\varphi\left(a_{0}, b\right)-k$, so $k^{\prime} \in \operatorname{int} C$. Since the function $\varphi(\cdot, b)$ is $C$-usc at $a_{0} \in A$, we obtain for $k^{\prime}$ that there exists a neighborhood $U_{a_{0}} \subset E$ of $a_{0}$ such that

$$
\begin{aligned}
\varphi(u, b) & \in \varphi\left(a_{0}, b\right)+k^{\prime}-\operatorname{int} C \\
& =\varphi\left(a_{0}, b\right)-\varphi\left(a_{0}, b\right)-k-\operatorname{int} C \\
& =-k-\operatorname{int} C, \text { for all } u \in U_{a_{0}} .
\end{aligned}
$$


Hence we have that $\varphi(u, b)+k \in-\operatorname{int} C$ for all $u \in U_{a_{0}}$, which means that $U_{b, k}$ is an open set.

Since the family $\left\{U_{b, k}\right\}$ is an open covering of the compact set $A$, we can select a finite subfamily which covers the same set $A$, i.e., there exist $b_{1}, \ldots, b_{n} \in B$ and $k_{1}, \ldots, k_{n} \in \operatorname{int} C$ such that

$$
A \subseteq \bigcup_{j=1}^{n} U_{b_{j}, k_{j}}
$$

For these $k_{1}, \ldots, k_{n} \in \operatorname{int} C$, we have that there exist $V_{1}, \ldots, V_{n}$ balanced neighborhoods of the origin of $Z$ such that $k_{j}+V_{j} \subset C$ for all $j \in\{1, \ldots, n\}$ (see e.g. [13]).

Define $V:=V_{1} \cap \cdots \cap V_{n}$, thus $\mathrm{V}$ is a balanced neighborhood of the origin of the space $Z$. Let $k_{0} \in V \cap \operatorname{int} C$, so we have $-k_{0} \in V$. Hence,

$$
k_{j}-k_{0} \in k_{j}+V \subseteq k_{j}+V_{j} \subseteq C, \text { for all } j \in\{1, \ldots, n\},
$$

which gives

$$
k_{j}-k_{0} \in C, \text { for all } j \in\{1, \ldots, n\} .
$$

Now define the vector-valued function $F: A \rightarrow Z^{n}$ by

$$
F(a):=\left(\varphi\left(a, b_{1}\right)+k_{0}, \ldots, \varphi\left(a, b_{n}\right)+k_{0}\right) .
$$

Assert that

$$
\operatorname{co} F(A) \cap(\operatorname{int} C)^{n}=\emptyset,
$$

where $\operatorname{co} F(A)$ denotes the convex hull of the set $F(A)$. Supposing the contrary, there exist $a_{1}, \ldots, a_{m} \in A$ and $\lambda_{1}, \ldots, \lambda_{m} \geq 0$ with $\sum_{i=1}^{m} \lambda_{i}=1$ such that $\sum_{i=1}^{m} \lambda_{i} F\left(a_{i}\right) \in(\operatorname{int} C)^{n}$, or equivalently,

$$
\sum_{i=1}^{m} \lambda_{i}\left[\varphi\left(a_{i}, b_{j}\right)+k_{0}\right] \in \operatorname{int} C, \text { for each } j \in\{1, \ldots, n\}, \text { which gives }
$$

$$
\sum_{i=1}^{m} \lambda_{i} \varphi\left(a_{i}, b_{j}\right)+k_{0} \in \operatorname{int} C, \text { for each } j \in\{1, \ldots, n\} .
$$

Let $u^{*} \in C^{*}$ be a nonzero functional for which (ii) holds. Applying $u^{*}$ to the relation above and taking into account Lemma 2.3 we obtain that

$$
\sum_{i=1}^{m} \lambda_{i} u^{*}\left(\varphi\left(a_{i}, b_{j}\right)\right)+u^{*}\left(k_{0}\right)>0 \text {. }
$$


Passing to the minimum over $j$ we have

$$
\min _{1 \leq j \leq n} \sum_{i=1}^{m} \lambda_{i} u^{*}\left(\varphi\left(a_{i}, b_{j}\right)\right)>-u^{*}\left(k_{0}\right),
$$

thus, assumption (ii) and relation (5) imply that

$$
\sup _{a \in A} \min _{1 \leq j \leq n} u^{*}\left(\varphi\left(a, b_{j}\right)\right)>-u^{*}\left(k_{0}\right) .
$$

For each $a \in A$, by relation (1) we have that there exists $j_{0} \in\{1, \ldots, n\}$ such that $a \in U_{b_{j_{0}}, k_{j_{0}}}$, i.e. $\varphi\left(a, b_{j_{0}}\right)+k_{j_{0}} \in-$ int $C$. This, together with (2) imply that

$$
\varphi\left(a, b_{j_{0}}\right)+k_{0} \in-k_{j_{0}}+k_{0}-\operatorname{int} C \subseteq-\operatorname{int} C .
$$

By Lemma 2.3 and using the fact that $u^{*} \in C^{*}$ we obtain that

$$
u^{*}\left(\varphi\left(a, b_{j_{0}}\right)\right)+u^{*}\left(k_{0}\right)<0 .
$$

Thus for each $a \in A$

$$
\min _{1 \leq j \leq n} u^{*}\left(\varphi\left(a, b_{j}\right)\right)<-u^{*}\left(k_{0}\right),
$$

and passing to supremum over $a$ we get a contradiction.

By the separation theorem of convex sets of Eidelheit (see for instance [13]), we have that there exists $z^{*} \in\left(Z^{n}\right)^{*}$ a nonzero functional such that

$$
z^{*}(u) \leq 0, \text { for all } u \in \operatorname{co} F(A) \text { and }
$$

$$
z^{*}(c) \geq 0, \text { for all } c \in(\operatorname{int} C)^{n} .
$$

Using the representation $z^{*}=\left(z_{1}^{*}, \ldots, z_{n}^{*}\right)$, by a standard argument we deduce that $z_{j}^{*} \in C^{*}$ for all $j \in\{1, \ldots, n\}$.

In particular, by (7), we have $z^{*}(u) \leq 0$ for all $u \in F(A)$. This means that for any $a \in A, z^{*}(F(a)) \leq 0$, or equivalently,

$$
\sum_{j=1}^{n} z_{j}^{*}\left(\varphi\left(a, b_{j}\right)+k_{0}\right) \leq 0 .
$$

Taking into account the linearity of $z_{j}^{*} \in C^{*}$ for all $j \in\{1, \ldots, n\}$, Lemma 2.3 and the fact that not all $z_{j}^{*}$ are zero we obtain

$$
\sum_{j=1}^{n} z_{j}^{*}\left(\varphi\left(a, b_{j}\right)\right) \leq-\sum_{j=1}^{n} z_{j}^{*}\left(k_{0}\right)<0 .
$$


Passing to supremum over $a \in A$ in the upper relation we deduce that

$$
\sup _{a \in A} \sum_{j=1}^{n} z_{j}^{*}\left(\varphi\left(a, b_{j}\right)\right)<0,
$$

which is a contradiction to assumption (iii). This completes the proof.

Assumption (ii) of Theorem 2.4 is a kind of generalized concavity of the function $\varphi$ in its first variable with respect to the cone $C$. To see this, we first recall the following convexity notion given by V. Jeyakumar in [9] for a vector-valued function and then adapt it for bifunctions. A function $f: A \rightarrow Z$ is said to be $C$-subconvexlike (see [9], [12]) if there exists $c \in \operatorname{int} C$ such that for all $\varepsilon>0$, $t \in[0,1]$ and $a_{1}, a_{2} \in A$ there exists $\bar{a} \in A$ such that

$$
f(\bar{a}) \leq_{C} t f\left(a_{1}\right)+(1-t) f\left(a_{2}\right)+\epsilon c .
$$

Lemma 2.5. [12]. Let $A \subseteq E$ be a nonempty set, $f: A \rightarrow Z$ a given function and $C$ a convex solid closed cone. The following assertions are equivalent:

(i) $f$ is $C$-subconvexlike on A;

(ii) there exists $c \in C$ such that for all $\varepsilon>0, t \in[0,1]$ and $a_{1}, a_{2} \in A$ there exists $\bar{a} \in A$ such that

$$
f(\bar{a}) \leq_{C} t f\left(a_{1}\right)+(1-t) f\left(a_{2}\right)+\epsilon c ;
$$

(iii) for each $l \in$ int $C, a_{1}, a_{2} \in A$ and $\lambda \in(0,1)$ there exists $\bar{a} \in A$ such that

$$
f(\bar{a}) \leq_{C} \lambda f\left(a_{1}\right)+(1-\lambda) f\left(a_{2}\right)+l .
$$

Definition 2.6. Let $\varphi: A \times B \rightarrow Z$ be a function. The function $\varphi$ is said to be:

(i) C-subconcavelike in its first variable if for each $l \in \operatorname{int} C, a_{1}, a_{2} \in A$ and $\lambda \in(0,1)$ there exists $\bar{a} \in A$ such that

$$
\varphi(\bar{a}, b) \geq_{C} \lambda \varphi\left(a_{1}, b\right)+(1-\lambda) \varphi\left(a_{2}, b\right)-l, \text { for all } b \in B .
$$

(ii) C-subconvexlike in its second variable if for each $l \in \operatorname{int} C, b_{1}, b_{2} \in A$ and $\lambda \in(0,1)$ there exists $\bar{b} \in A$ such that

$$
\varphi(a, \bar{b}) \leq_{C} \lambda \varphi\left(a, b_{1}\right)+(1-\lambda) \varphi\left(a, b_{2}\right)+l, \text { for all } a \in A .
$$

(iii) $\mathrm{C}$-subconcavelike-subconvexlike on $A \times B$ if it is $\mathrm{C}$-subconcavelike in its first variable and $\mathrm{C}$-subconvexlike in its second variable. 
In case $Z=\mathbb{R}$ and $C=\mathbb{R}_{+}$we use the terms subconcavelike, subconvexlike and subconcavelike-subconvexlike for $\mathbb{R}_{+}$-subconcavelike, $\mathbb{R}_{+}$-subconvexlike and $\mathbb{R}_{+}$-subconcavelike-subconvexlike, respectively.

It is easy to check by induction that the concept of $C$-subconcavelikeness can be characterized as follows.

Proposition 2.7. The function $\varphi: A \times B \rightarrow Z$ is $C$-subconcavelike in its first variable if and only if for each $l \in$ int $C, a_{1}, \ldots, a_{m} \in A, \lambda_{1}, \ldots, \lambda_{m} \geq 0$ with $\sum_{i=1}^{m} \lambda_{i}=1$ there exists $\bar{a} \in A$ such that

$$
\varphi(\bar{a}, b) \geq_{C} \sum_{i=1}^{m} \lambda_{i} \varphi\left(a_{i}, b\right)-l, \text { for all } b \in B .
$$

Using Proposition 2.7 we obtain by Theorem 2.4 the following result.

Corollary 2.8. Let $A$ be a compact set and let $\varphi: A \times B \rightarrow Z$ be a function such that

(i) $\varphi$ is $C$-usc and $C$-subconcavelike in its first variable;

(ii) for each $b_{1}, \ldots, b_{n} \in B, z_{1}^{*}, \ldots, z_{n}^{*} \in C^{*}$ not all zero one has

$$
\sup _{a \in A} \sum_{j=1}^{n} z_{j}^{*}\left(\varphi\left(a, b_{j}\right)\right) \geq 0 \text {. }
$$

Then the equilibrium problem (WVEP) admits a solution.

Proof. It is enough to show that assumption (ii) of Theorem 2.4 is satisfied. Let us prove that the $C$-subconcavelikeness of the function $\varphi$ implies assumption (ii) of the above theorem.

Fix $a_{1}, \ldots, a_{m} \in A, b_{1}, \ldots, b_{n} \in B, \lambda_{1}, \ldots, \lambda_{m} \geq 0$ with $\sum_{i=1}^{m} \lambda_{i}=1$, and $u^{*} \in C^{*} \backslash\{0\}$.

By the C-subconcavelikeness of $\varphi$ in its first variable, for each $l \in \operatorname{int} C$ there exists $\bar{a} \in A$ such that

$$
\sum_{i=1}^{m} \lambda_{i} \varphi\left(a_{i}, b_{j}\right) \leq_{C} \varphi\left(\bar{a}, b_{j}\right)+l, \text { for each } j \in\{1, \ldots, n\} .
$$

Applying $u^{*}$, relation (9) becomes

$$
\sum_{i=1}^{m} \lambda_{i} u^{*} \varphi\left(a_{i}, b_{j}\right) \leq u^{*}\left(\varphi\left(\bar{a}, b_{j}\right)\right)+u^{*}(l), \text { for each } j \in\{1, \ldots, n\},
$$


which, by passing to minimum over $j$ yields

$$
\begin{aligned}
\min _{1 \leq j \leq n} \sum_{i=1}^{m} \lambda_{i} u^{*}\left(\varphi\left(a_{i}, b_{j}\right)\right) & \leq \min _{1 \leq j \leq n} u^{*}\left(\varphi\left(\bar{a}, b_{j}\right)\right)+u^{*}(l) \\
& \leq \sup _{a \in A} \min _{1 \leq j \leq n} u^{*}\left(\varphi\left(a, b_{j}\right)\right)+u^{*}(l) .
\end{aligned}
$$

Since this relation holds for each $l \in \operatorname{int} C$ we obtain assumption (ii) of Theorem 2.4. Hence (WVEP) admits a solution.

In what follows we deal with the scalar case. Let $Z=\mathbb{R}$ and $C=C^{*}=\mathbb{R}_{+}$. Then our vector equilibrium problem (WVEP) becomes

(EP) find $\bar{a} \in A$ such that $\varphi(\bar{a}, b) \geq 0$, for all $b \in B$.

Theorem 2.4 permits us to reobtain an earlier result of existence of solutions for (EP).

Corollary 2.9. [10]. Let $A$ be a compact set, let $B$ be a nonempty set and let $\varphi: A \times B \rightarrow \mathbb{R}$ be a function such that

(i) for each $b \in B$, the function $\varphi(\cdot, b): A \rightarrow \mathbb{R}$ is usc on $A$;

(ii) for each $a_{1}, \ldots, a_{m} \in A, \lambda_{1}, \ldots, \lambda_{m} \geq 0$ with $\sum_{i=1}^{m} \lambda_{i}=1, b_{1}, \ldots, b_{n} \in B$

$$
\min _{1 \leq j \leq n} \sum_{i=1}^{m} \lambda_{i} \varphi\left(a_{i}, b_{j}\right) \leq \sup _{a \in A} \min _{1 \leq j \leq n} \varphi\left(a, b_{j}\right)
$$

(iii) for each $b_{1}, \ldots, b_{n} \in B, \mu_{1}, \ldots, \mu_{n} \geq 0$ with $\sum_{j=1}^{n} \mu_{j}=1$

$$
\sup _{a \in A} \sum_{j=1}^{n} \mu_{j} \varphi\left(a, b_{j}\right) \geq 0 .
$$

Then the equilibrium problem (EP) admits a solution.

Proof. We show that the assumptions of Theorem 2.4 are satisfied. It is obvious that conditions (i) and (ii) of Theorem 2.4 are satisfied (the latter with $u^{*}=1$ ).

Let $b_{1}, \ldots, b_{n} \in B, z_{1}^{*}, \ldots, z_{n}^{*} \in \mathbb{R}_{+}$not all zero and denote $\mu_{j}:=\frac{z_{j}^{*}}{\nu}$ for all $j \in\{1, \ldots, n\}$, where $\nu=\sum_{j=1}^{n} z_{j}^{*}$. Thus each $\mu_{j} \geq 0$ and $\sum_{j=1}^{n} \mu_{j}=1$. Hence by assumption (iii) we obtain

$$
\frac{1}{\nu} \sup _{a \in A} \sum_{j=1}^{n} z_{j}^{*} \varphi\left(a, b_{j}\right) \geq 0,
$$


i.e., assumption (iii) of Theorem 2.4. Thus the result follows by Theorem 2.4.

By Corollary 2.9 we can deduce a sufficient condition for (EP) using Definition 2.6 for a real-valued function.

Corollary 2.10. Let $A$ be a compact set and let $\varphi: A \times B \rightarrow \mathbb{R}$ be a function such that:

(i) for each $b \in B$, the function $\varphi(\cdot, b): A \rightarrow \mathbb{R}$ is usc on $A$;

(ii) $\varphi$ is subconcavelike-subconvexlike on $A \times B$;

(iii) $\sup _{a \in A} \varphi(a, b) \geq 0$, for each $b \in B$.

Then the equilibrium problem $(E P)$ admits a solution.

Proof. It is obvious that assumption (i) of Corollary 2.9 is satisfied.

Also, following the same line of proof as in Corollary 2.8, it is easy to check that assumption (ii) of Corollary 2.9 is satisfied.

Now, let $b_{1}, \ldots, b_{n} \in B$ and $\mu_{1}, \ldots, \mu_{n} \geq 0$ with $\sum_{j=1}^{n} \mu_{j}=1$. By the subconvexlikeness of function $\varphi$ in its second variable, for each $\epsilon>0$, there exists $\bar{b} \in B$, such that

$$
\varphi(a, \bar{b}) \leq \sum_{j=1}^{n} \mu_{j} \varphi\left(a, b_{j}\right)+\epsilon, \text { for all } a \in A .
$$

Since the relation

$$
0 \leq \sup _{a \in A} \varphi(a, \bar{b}) \leq \sup _{a \in A} \sum_{j=1}^{n} \mu_{j} \varphi\left(a, b_{j}\right)+\epsilon,
$$

is satisfied for each $\epsilon>0$, we have that assumption (iii) of Corollary 2.9 is verified.

\section{Applications to Minimization Problems}

Let $S \subseteq Z$. In what follows we shall denote by $\operatorname{Min}_{w} S$ the set of weak minima of $S$ with respect to the cone $C$, i.e., $z_{0} \in \operatorname{Min}_{w} S$ means $z_{0} \in S$ and

$$
S \cap\left(z_{0}-\operatorname{int} C\right)=\emptyset .
$$

Similarly, the set $\operatorname{Max}_{w} S$ denotes the set of weak maxima of $S$ with respect to the cone $C$, i.e., $z_{0} \in \operatorname{Max}_{w} S$ means $z_{0} \in S$ and

$$
S \cap\left(z_{0}+\operatorname{int} C\right)=\emptyset .
$$


Let $l(C)=C \cap(-C)$. In what follows consider the vectors $e \in C$ and $e^{*} \in C^{*}$ satisfying the assumption

(D) $\quad e \notin l(C)$ and $\left\langle e^{*}, e\right\rangle=1$.

Let $F: A \rightarrow Z$ be fixed. For every $e, e^{*}$ satisfying (D) consider the scalar equilibrium problem:

$$
(\mathrm{EP})_{e, e^{*}} \quad \text { find } \bar{a} \in A \text { such that } f(\bar{a}, b) \geq 0, \text { for all } b \in A \text {, }
$$

where $f: A \times A \rightarrow \mathbb{R}$ is given by $f(a, b)=\left\langle e^{*}, F(b)-F(a)\right\rangle$.

Denoting by $\varphi: A \times A \rightarrow Z$ the vector function defined by $\varphi(a, b):=F(b)-$ $F(a)$, it is easy to see that (WVEP) for this $\varphi$ becomes the weak vector minimization problem:

(WVMP) find $\bar{a} \in A$ such that $F(b)-F(\bar{a}) \notin-\operatorname{int} C$, for all $b \in A$.

The point $\bar{a}$ is a solution of (WVMP) if and only if $F(\bar{a}) \in \operatorname{Min}_{w} F(A)$. The next statement is straightforward.

Proposition 3.1. For every $e$ and $e^{*}$ satisfying assumption $(D)$, the set of solutions of $(E P)_{e, e^{*}}$ is contained in the set of solutions of $(W V M P)$.

Proof. Let $\bar{a}$ be a solution of $(E P)_{e, e^{*}}$. Then we have

$$
\left\langle e^{*}, F(b)-F(\bar{a})\right\rangle \geq 0, \text { for all } b \in A,
$$

which implies that $F(b)-F(\bar{a}) \notin-\operatorname{int} C$. Hence $\bar{a}$ is a solution for the problem (WVMP).

Observe that the reverse implication in Proposition 3.1 is not true in general, as the following example shows.

Example 3.2. Let $F: \mathbb{R} \rightarrow \mathbb{R}^{2}$ be the vector-valued function defined by

$$
F(a)=\left\{\begin{array}{l}
\left(-1, \frac{1}{|a|}\right), \text { if } a \neq 0 \\
(0,0), \text { if } a=0 .
\end{array}\right.
$$

Take $e=e^{*}=(1,0)$ and $C=\mathbb{R}_{+}^{2}$. We have

$$
f(0, b)=\langle(1,0), F(b)\rangle=\left\{\begin{array}{l}
-1, \text { if } b \neq 0 \\
0, \text { if } b=0
\end{array}\right.
$$


thus 0 is not a solution of the scalar equilibrium problem $(E P)_{e, e^{*}}$.

We verify if $a=0$ is a solution of (WVMP). Indeed,

$$
\varphi(0, b)=F(b)-F(0)=\left\{\begin{array}{l}
\left(-1, \frac{1}{|b|}\right), \text { if } b \neq 0 \\
(0,0), \text { if } b=0 .
\end{array}\right.
$$

This relation shows that $\varphi(0, b) \notin-\operatorname{int} C$ for each $b \in \mathbb{R}$, which implies that $a=0$ is a solution of (WVMP).

It is interesting to notice that in the previous example every $a \neq 0$ is a solution of $(E P)_{e, e^{*}}$. Indeed,

$$
f(a, b)=\langle(1,0), F(b)-F(a)\rangle=\left\{\begin{array}{l}
0, \text { if } b \neq 0 \\
1, \text { if } b=0,
\end{array}\right.
$$

hence $f(a, b) \geq 0$ for all $b \in \mathbb{R}$, i.e. $a \neq 0$ is a solution of $(E P)_{e, e^{*}}$. By Proposition 3.1 it follows that each real number is a solution of (WVMP).

The next result, well-known in vector optimization, is a consequence of Corollary 2.9 .

Proposition 3.3. If $F$ is $C$-lsc on a compact set $A$, then the scalar equilibrium problem $(E P)_{e, e^{*}}$ admits a solution for every $e, e^{*}$ satisfying $(D)$.

Proof. We show that the assumptions of Corollary 2.9 are satisfied.

Fix $b \in A, \epsilon>0$ and let $a \in A$. Then by Lemma 2.3 we have that there exists $k \in \operatorname{int} C$ such that $\epsilon=\left\langle e^{*}, k\right\rangle$. For this $k$ we obtain by the definition of the $C$-lsc of $F$ that there exists a neighborhood $U$ of $a$ such that

$$
F(u)-F(a)+k \in \operatorname{int} C, \text { for all } u \in U .
$$

This is equivalent to

$$
-[F(b)-F(u)]+[F(b)-F(a)]+k \in \operatorname{int} C, \text { for all } u \in U .
$$

By applying $e^{*}$ to the above relation and by Lemma 2.3 we obtain

$$
\left\langle e^{*}, \varphi(a, b)-\varphi(u, b)\right\rangle+\left\langle e^{*}, k\right\rangle>0, \text { for all } u \in U,
$$

giving that

$$
f(a, b)-f(u, b)>-\epsilon, \text { for all } u \in U,
$$

which is equivalent to

$$
f(u, b)-f(a, b)<\epsilon, \text { for all } u \in U .
$$


Hence, we have obtained that $f(\cdot, b)$ is usc for each $b \in A$ and assumption (i) of Corollary 2.9 is satisfied.

To verify assumption (ii) of Corollary 2.9 , choose $a_{1}, \ldots, a_{m} \in A, \lambda_{1}, \ldots, \lambda_{m} \geq$ 0 with $\sum_{i=1}^{m} \lambda_{i}=1$ and $b_{1}, \ldots, b_{n} \in B$. Then the relation

$$
\min _{1 \leq j \leq n} \sum_{i=1}^{m} \lambda_{i} f\left(a_{i}, b_{j}\right) \leq \sup _{a \in A} \min _{1 \leq j \leq n} f\left(a, b_{j}\right)
$$

becomes

$$
\min _{1 \leq j \leq n} \sum_{i=1}^{m} \lambda_{i}\left\langle e^{*}, F\left(b_{j}\right)-F\left(a_{i}\right)\right\rangle \leq \sup _{a \in A} \min _{1 \leq j \leq n}\left\langle e^{*}, F\left(b_{j}\right)-F(a)\right\rangle .
$$

Taking into account that $\sum_{i=1}^{m} \lambda_{i}=1$ and the linearity of $e^{*}$ we obtain

$$
\min _{1 \leq j \leq n}\left\langle e^{*}, F\left(b_{j}\right)\right\rangle-\left\langle e^{*}, \sum_{i=1}^{m} \lambda_{i} F\left(a_{i}\right)\right\rangle \leq \min _{1 \leq j \leq n}\left\langle e^{*}, F\left(b_{j}\right)\right\rangle+\sup _{a \in A}\left(-\left\langle e^{*}, F(a)\right\rangle\right) .
$$

This implies

$$
\inf _{a \in A}\left\langle e^{*}, F(a)\right\rangle \leq\left\langle e^{*}, \sum_{i=1}^{m} \lambda_{i} F\left(a_{i}\right)\right\rangle,
$$

relation that is satisfied by any function $F$. So, $F$ satisfies assumption (ii).

Finally, let $b_{1}, \ldots, b_{n} \in A, \mu_{1}, \ldots, \mu_{n} \geq 0$ not all zero with $\sum_{j=1}^{n} \mu_{j}=1$.

Relation (iii) of Corollary 2.9 is equivalent to

$$
\sup _{a \in A} \sum_{j=1}^{n} \mu_{j}\left\langle e^{*}, F\left(b_{j}\right)-F(a)\right\rangle \geq 0,
$$

or

$$
\sup _{a \in A}\left[\left\langle e^{*}, \sum_{j=1}^{n} \mu_{j} F\left(b_{j}\right)\right\rangle-\left\langle e^{*}, F(a)\right\rangle\right] \geq 0 .
$$

This relation can be written as

$$
\inf _{a \in A}\left\langle e^{*}, F(a)\right\rangle \leq\left\langle e^{*}, \sum_{j=1}^{n} \lambda_{j} F\left(a_{j}\right)\right\rangle
$$

and it is verified by any function $F$.

Therefore, by Corollary 2.9, we have that $(E P)_{e, e^{*}}$ has a solution. 
Notice that in vector optimization different kind of lower semicontinuity concepts have been used. The next concept introduced in [6] and [15] is a slight relaxation of lower semicontinuity.

Definition 3.4. The function $f: X \rightarrow Z$ is said to be quasi-lower semicontinuous at $x_{0} \in X$ (q-1sc in short) if for each $b \in Z$ such that $b \geq_{C} f\left(x_{0}\right)$, there exists a neighborhood $U$ of $x_{0}$ such that $b \nsupseteq C f(u)$ for each $u \in U$.

The question whether the $C$-lsc in Proposition 3.3 can be weakened to $q$-lsc arises naturally. The next example shows that the answer is negative.

Example 3.5. Let $Z=\mathbb{R}^{2}, C=\mathbb{R}_{+}^{2}$, and $F:[0,1] \rightarrow \mathbb{R}^{2}$ defined by

$$
F(t)=\left\{\begin{array}{l}
\left(-\frac{2}{t}, \frac{1}{t}\right), \text { if } t \neq 0 \\
(0,0), \text { if } t=0
\end{array}\right.
$$

It is easy to verify that $F$ is q-1sc at 0 but not $\mathbb{R}_{+}^{2}-1$ sc at 0 .

For $e=e^{*}=\left(\frac{1}{\sqrt{2}}, \frac{1}{\sqrt{2}}\right) \in$ int $\mathbb{R}_{+}^{2}$, we have that $e^{*}(e)=1$ and $(E P)_{e, e^{*}}$ does not admit any solution.

If $a \neq 0$, then

$$
f(a, b)=\left\langle\left(\frac{1}{\sqrt{2}}, \frac{1}{\sqrt{2}}\right), F(b)-F(a)\right\rangle=\left\{\begin{array}{l}
\frac{1}{\sqrt{2}}\left(-\frac{1}{b}+\frac{1}{a}\right), \text { if } b \neq 0 \\
\frac{1}{\sqrt{2} a}, \text { if } b=0,
\end{array}\right.
$$

therefore $f(a, b)<0$ for all $b<a$. Therefore, $a \neq 0$ is not a solution of $(E P)_{e, e^{*} \text {. }}$

For $a=0$, the function $f$ becomes

$$
f(0, b)=\left\langle\left(\frac{1}{\sqrt{2}}, \frac{1}{\sqrt{2}}\right), F(b)-F(0)\right\rangle=\left\{\begin{array}{l}
-\frac{1}{\sqrt{2} b}, \text { if } b \neq 0 \\
0, \text { if } b=0 .
\end{array}\right.
$$

We observe that for each $b \in(0,1], f(0, b)<0$. This means that $a=0$ is not a solution of $(E P)_{e, e^{*}}$. By consequence, $(E P)_{e, e^{*}}$ has no solutions, although the set of solutions of (WVMP) is $[0,1]$.

Proposition 3.6. If $F$ is $C$-lsc on a compact set $A$, then the weak vector minimization problem (WVMP) admits a solution.

\section{Applications to Cone-Saddle Points}

Let $X$ and $Y$ be nonempty subsets of two topological spaces and $f: X \times Y \rightarrow Z$. For $x \in X$ and $y \in Y$ we consider the sets 


$$
f(x, Y):=\{f(x, y) \mid y \in Y\} \text { and } f(X, y):=\{f(x, y) \mid x \in X\} .
$$

We recall the following concept which extends the classical definition of a saddle point for scalar functions.

Definition 4.1. [14] A point $\left(x_{0}, y_{0}\right) \in X \times Y$ is said to be a weak C-saddle point of $\mathrm{f}$ on $X \times Y$ if

$$
f\left(x_{0}, y_{0}\right) \in \max _{w} f\left(X, y_{0}\right) \cap \min _{w} f\left(x_{0}, Y\right) .
$$

Proposition 4.2. Let $A=B=X \times Y$ and the vector function $\varphi: A \times B \rightarrow Z$ defined by $\varphi(a, b)=f(x, v)-f(u, y)$, for all $a=(x, y)$ and $b=(u, v) \in X \times Y$. If $\bar{a}$ is a solution for (WVEP) then $\bar{a}$ is a weak $C$-saddle point of $f$.

Proof. Let $\bar{a}=(\bar{x}, \bar{y})$ be a solution of the problem (WVEP). Then

$$
\varphi(\bar{a}, b) \notin-\text { int } C \text {, for all } b \in B \text {. }
$$

This implies that $f(\bar{x}, v)-f(u, \bar{y}) \notin-$ int $C$ for all $(u, v) \in X \times Y$. If we take $u:=\bar{x}$ and $v:=y$ we obtain

$$
f(\bar{x}, y) \notin f(\bar{x}, \bar{y})-\operatorname{int} C, \text { for all } y \in Y,
$$

which leads to $f(\bar{x}, \bar{y}) \in \operatorname{Min}_{w} f(\bar{x}, Y)$.

Let $u:=x$ and $v:=\bar{y}$. Then we have

$$
f(x, \bar{y}) \notin f(\bar{x}, \bar{y})+\text { int } C, \text { for all } x \in X,
$$

which gives us that $f(\bar{x}, \bar{y}) \in \operatorname{Max}_{w} f(X, \bar{y})$.

Relations (11) and (12) imply that $(\bar{x}, \bar{y})$ is a weak $C$-saddle-point of $f$.

Theorem 4.3. Let $X$ and $Y$ be compact sets and $f: X \times Y \rightarrow Z$ be a function. If:

(i) $f$ is $C$-usc with respect to its first variable and $C$-lsc with respect to its second variable;

(ii) $f$ is $C$-subconcavelike-subconvexlike on $X \times Y$, then $f$ admits a weak $C$-saddle-point.

Proof. Let $e \in C \backslash l(C)$. Then there exists $e^{*} \in C^{*}$ such that $\left\langle e^{*}, e\right\rangle=1$. We define the sets $A=B:=X \times Y$ and the function $\varphi: A \times B \rightarrow \mathbb{R}$, by

$$
\varphi(a, b):=\left\langle e^{*}, f(x, v)-f(u, y)\right\rangle, \text { for all } a=(x, y) \in A \text { and } b=(u, v) \in B \text {. }
$$

It is easy to check that the assumptions of Corollary 2.10 are satisfied for this function. By this corollary, Propositions 3.1 and 4.2 we obtain that $f$ admits a weak $C$-saddle point. 


\section{ACKNOWLEDGMENTS}

The authors are indebted to an anonymous reviewer for valuable suggestions and comments which improved the quality of the paper.

\section{REFERENCES}

1. M. Bianchi and R. Pini, A note on equilibrium problems with properly quasimonotone bifunctions, J. Global Optim., 20 (2001), 67-76.

2. M. Bianchi and R. Pini, Coercivity conditions for equilibrium problems, J. Optim. Theory Appl., 124 (2005), 79-92.

3. M. Bianchi and S. Schaible, Generalized monotone bifunctions and equilibrium problems, J. Optim. Theory Appl., 90 (1996), 31-43.

4. M. Bianchi, G. Kassay and R. Pini, Ekeland's principle for vector equilibrium problems, Nonlinear Analysis, 66 (2007), 1454-1464.

5. E. Blum and W. Oettli, From optimization and variational inequalities to equilibrium problems, Math. Stud., 63 (1994), 123-145.

6. J. Borwein, J. Penot and M. Thera, Conjugate convex operators, J. Math. Anal. Appl., 102 (1984), 339-414.

7. Dinh The Luc, Lecture Notes in Economics and Mathematical Systems, Theory of Vector Optimization, Springer-Verlag, Berlin, 1989.

8. A. N. Iusem, G. Kassay and W. Sosa, On certain conditions for the existence of solutions of equlibrium problems, Mathematical Programming, Ser. B, 116 (2009), 259-273.

9. V. Jeyakumar, A generalization of a minimax theorem of Fan via a theorem of the alternative, Journal of Optimization Theory and Applications, 48(3) (1986), 525-533.

10. G. Kassay and J. Kolumbán, On a generalized sup-inf problem, J. Optim. Theory Appl., 91 (1996), 651-670.

11. G. Kassay, The equilibrium problem and related topics, Risoprint Cluj-Napoca, 2000.

12. S. Paeck, Convexlike and concavelike conditions in alternative, minimax, and minimization theorems, Journal of Optimization Theory and Applications, 74(2) (1992), 317-332.

13. W. Rudin, Functional Analysis, McGraw-Hill, 1973.

14. T. Tanaka, Generalized semicontinuity and existence theorems for cone saddle points, Appl. Math. Optim., 36(3) (1997), 313-322.

15. M. Thera, Études des fonctions convexes vectorielles semi-continues, Thèse de $3^{\mathrm{e}}$ cycle, Université de Pau, 1978. 
Adela Capătă and Gábor Kassay

Department of Mathematics,

Babeş-Bolyai University,

M. Kogălniceanu, no. 1,

400084 Cluj-Napoca,

Romania

E-mail: adela.capata@math.ubbcluj.ro kassay@math.ubbcluj.ro 